\title{
LAST PLANNER SYSTEM - THE NEED FOR NEW METRICS
}

\author{
Ghali El Samad ${ }^{1}$, Farook R. Hamzeh ${ }^{2}$, and Samir Emdanat ${ }^{3}$
}

\begin{abstract}
Several metrics have been used to evaluate the planning performance within the Last Planner System (LPS). Percent Planned Complete (PPC), which measures the reliability of weekly work planning, is the most commonly used metric. However, studies have shown the need to complement PPC with other metrics to measure performance. Researchers have developed many metrics to assess the makeready process, workflow reliability, and weekly work planning. Many of those metrics were either inconsistently used, showed no correlation with the overall project performance, or required data that was too difficult and time-consuming to collect. This paper offers an overview of the various metrics proposed in the literature. It also proposes new metrics and details their calculation method to measure aspects not yet supported by a measurement metric. This paper is useful for last planners who can employ the newly suggested metrics to assess weekly work planning performance taking into account activity characteristics.
\end{abstract}

Keywords: Last Planner ${ }^{\circledR}$ System, Workflow, Planning Reliability, Metrics

\section{INTRODUCTION}

The Last Planner System (LPS) is widely used in construction projects to improve workflow and increase the reliability of construction planning. LPS acknowledges the shortcomings of forecasts and thus recommends planning in greater depth as the team gets closer to completing the work. It is best described as a mechanism for transforming the work that should be done into what can be done, forming an inventory of work madeready from which the Last Planners commit to what they will do (Ballard 2000).

LPS consists of four planning stages: (1) Master Scheduling: Summarizes all the work that should be done in abstract terms. (2) Phase Scheduling: Defines project phases and is used to coordinate actions that extend beyond the lookahead window. Phase schedules provide more details regarding what should be done and when. (3) Lookahead Planning: Presents a time frame of roughly two to six weeks and is the stage where tasks are broken down and made ready. (4) Commitment Planning: Indicates the most detailed planning stage that results in commitments to deliver the work that was placed on the Weekly Work Plan (WWP) (Ballard 2000; Tommelein and Ballard 1997).

Many researchers and practitioners have developed metrics to measure the planning performance when applying the LPS in addition to PPC. Some metrics measure the success of the lookahead stage at anticipating tasks and removing constraints to make activities ready for implementation. Others measure productivity and progress both at the project level and the weekly work plan level. More metrics were proposed to align long-term

1 Graduate Student, Civil and Environmental Engineering Department, American University of Beirut, Lebanon, ghe02@mail.aub.edu

2 Assistant Professor, Civil and Environmental Engineering Department, American University of Beirut, Lebanon,+961350000 ext 3616, fax:+961 1 744462, farook.hamzeh@aub.edu.lb

3 Director of Management Services, Ghafari Associates, LLC, 17101 Michigan Avenue, Dearborn, Michigan 48126, semdanat@ghafari.com 
planning with short-term planning (Ballard 2000; Chitla and Abdelhamid 2003; Emdanat and Azambuja 2016; Gonzalez et al. 2008; Hamzeh et al. 2008; Hamzeh et al. 2015a; Hamzeh et al. 2015b; Jang and Kim 2007; Mitropoulos 2005).

This paper presents a review of all current LPS related metrics. To address aspects of production planning that are not addressed by current metrics, this paper proposes new metrics to assess weekly work planning performance and overall workflow. The suggested metrics complement PPC which naturally assumes that all activities are of equal value and importance. The proposed WWP metrics take into consideration activities status (Required, Not Required, Backlog, and New). Required activities are critical activities in the traditional Critical Path Method (CPM) understanding. Backlog activities are excess tasks that are ready but not necessary and which are to be executed in case of available capacity. New activities are tasks added to the WWP that were not foreseen in the lookahead process (Rouhana and Hamzeh 2016). The proposed workflow metrics considers the volume of the activity and the number and duration of its successor tasks.

\section{LPS METRICS}

Researchers and practitioners have developed several metrics for use within the LPS environment. This section presents an overview of most metrics discussed in the literature.

As previously mentioned, PPC is the most commonly used metric. The common understanding is that successful weekly work planning and a good implementation of LPS is linked to a high PPC. The metric is a "post production" measure of the reliability of weekly work planning. PPC is defined as the percentage of tasks completed at the end of a short future time period in comparison to the tasks that were promised to be completed at the beginning of that period (Ballard 2000). It is wrongly believed that having a high PPC value must result in shorter project durations. Other metrics are necessary to complement the PPC and measure the overall reliability of the lookahead process and the fulfilment of target milestones.

$$
P P C=\text { Did } / \text { Will }
$$

To align the weekly work plan assignments with the lookahead plan, Tasks Anticipated (TA) and Tasks Made Ready (TMR) can be used. Ballard first proposed the two metrics, initially called "Assignments Anticipated" and "Assignments Made Ready", in 1997, to evaluate the lookahead planning process. TA measures the performance of the lookahead planning process in anticipating tasks that will be committed to, for instance, two or three weeks in the future. On the other hand, TMR measures the ability of lookahead planning to make tasks ready for execution. It is the ratio of tasks with all their constraints removed in a certain time interval (usually two to three weeks) preceding execution to the tasks that were anticipated along the lookahead plan (Ballard 1997; Hamzeh and Aridi 2013). High values for TA and TMR can indicate shorter project durations (Hamzeh et al. 2015a; Hamzeh et al. 2015b).

$$
\begin{gathered}
T M R=\mathrm{Did} / \mathrm{Can} \\
T A=\text { Will/Can }
\end{gathered}
$$

Planned Work Ready (PWR) is a metric that assesses the quality of the lookahead process. PWR was proposed by Mitropoulos to indicate the percentage of work in the lookahead plan that is expected to be ready as planned in the lookahead horizon. The PWR metric acts as a forecast, and, it can provide a better evaluation of schedule performance when it is complemented with PPC (Mitropoulos 2005). 


$$
P W R=\frac{\text { Work Expected to be Performed in Lookahead }}{\text { Work that Should be Performed in Lookahead }}
$$

Mitropoulos (2005) also tried to measure the make ready process by suggesting three deltas: Delta_1 measures the constraints that prevent planned tasks from being ready. Delta_2 is the ratio of constraints removed compared to those that were expected to be removed. Finally, Delta_3 is the ratio of new constraints that were undetected during planning compared to the constraints identified. However, it is not easy to use the three deltas in assessing the make ready process since constraint analysis depends on the type of project and tends to differ from company to company.

$$
\begin{gathered}
\Delta_{1}=(\text { Constraints Promised to be Removed }) /(\text { Constraints Identified }) \\
\Delta_{2}=(\text { Constraints Removed }) /(\text { Constraints Promised to be Removed }) \\
\Delta_{3}=(\text { New Constraints }) /(\text { Constraints Identified })
\end{gathered}
$$

Jang and Kim (2007) proposed an additional metric, Percent of Constraint Removal (PCR), to measure the performance of the make ready process. PCR compares the number of constraint-free tasks when scheduling the WWP to the number of all planned tasks found on the lookahead plan. This metric is considered a leading indicator of workflow predictability and thus is calculated before the weekly work plan starts. Authors who have proposed this metric have successfully indicated that PCR and PPC are correlated (Jang and Kim 2008).

$$
\text { PCR }=\frac{\text { Number of Constraint Free Tasks When Scheduling WWP }}{\text { Number of Planned Tasks at Lookahead Plan }}=\frac{\text { Ready }}{\text { Can }}
$$

Furthermore, Alarcón et al. (2014) analyzed PPC, Schedule Performance Index (SPI), PCR, and schedule progress curves for 'successful' and 'unsuccessful' projects. The success of the projects used was determined based on their schedule performance. The results showed that an increase in planning reliability (PPC and PCR) could improve project progress. It was also observed that for successful projects, it is not enough to have high values for the metrics, for it is important as well to control their variability.

In contrast, some researchers tried to correlate workflow reliability to productivity. Ballard and Howell (1998) used the Performance Factor (PF) as an indirect measure to show the impact of LPS on productivity. PF is the ratio of actual to earned productivity and is usually represented in labor hours. However, no statistical relationship was found between PF and PPC (Gonzalez et al. 2008).

$$
P F=\frac{\text { Actual Labor Hours }}{\text { Earned Labor Hours }}
$$

Chitla and Abdelhamid (2003) investigated the difference between improvements based on PPC vs those that are based primarily on the Labor Utilization Factor (LUF). They expressed LUF in terms of productive and non-productive time and found that an increase in PPC causes an increase in LUF. However, an increase in LUF does not result in an increase in PPC. They recommend project managers to focus on PPC instead of wasting resources on improving LUF as LUF is only a measure of local production activation.

$$
L U F=\frac{\text { Effective Work }+\frac{1}{4} \text { Essential Contributory Work }}{\text { Effective }+ \text { Essential Contributory }+ \text { not useful }}
$$

Gonzalez et al. (2008) reformulated LPS metrics in order to be able to compare them with activity level performance indicators. For this, they proposed two metrics: Project 
Productivity Index (PPI) and Process Reliability index (PRI). PPI is considered an aggregate productivity index that overcomes PF limitations. It is calculated as the average of the Activity Productivity Indexes (API) where API is the ratio of average labor productivity to maximum labor productivity. PPI can reflect real productivity improvements because it is computed from maximum productivity on-site and not from expected productivity as is the case with PF. PPI has a good correlation with PPC. The other proposed metric, PRI, is a planning reliability index at activity level and is measured as the ratio of actual to planned weekly progress of a certain activity. Consequently, PRI was found to overcome PPC limitations for analyzing LPS effects at the activity level.

$$
\begin{gathered}
P P I=\left(\sum A P I / N\right) \times 100 \\
P R I=(A P / P P) \times 100
\end{gathered}
$$

Emdanat and Azambuja (2016) proposed three additional metrics to complement PPC, TA, and TMR in aligning short and long term planning. The first metric, Commitment Level (CL) is the percentage of the total committed required/critical activities with respect to the total required activities for any given work plan cycle. Fluctuations in CL were found to be correlated with an increase in late paths and decrease in float.

$$
C L=\text { Required Will/Should }
$$

Other metrics proposed were Percent Required Completed or Ongoing (PRCO) and Milestone Variance (MV). PRCO is the percentage of required/critical activities that are completed or expected to be completed on or before their promised completion dates with respect to the total required activities on the work plan. On the other hand, MV is the difference in days between the forecasted date to complete all remaining activities and the required date of the milestone. Emdanat and Azambuja (2016) demonstrated that teams who re-plan to maintain CL, PRCO, and PPC had a lower overall MV and hence were more reliable.

$$
\text { PRCO }=\frac{\text { Required completed }+ \text { Required Ongoing on Track }}{\text { Required Will }}
$$

Finally, Priven et al. (2014) developed a Lean Workflow Index (LWI) to describe workflow. LWI is a polynomial function that uses multiple location-based scheduling parameters including: (A) the product of the root mean squares of all flowlines, (C) the percentage of time with no breaks after finishing a floor, (D) the percentage of time crews are working, (E) work in progress, and (F) work out of sequence. The weight of each parameter was calibrated by using goal-seeking algorithms based on subjective survey results of Location Based Management Schedules. No correlation was found between LWI and PPC. Yet, LWI can be used to achieve a smooth workflow alongside PPC.

$$
L W I(t)=7 \% x A^{2}+33 \% x C^{2}+4 \% x D^{2}+31 \% x E^{2}+25 \% x F^{2}
$$

\section{Suggested Metrics}

Many metrics outlined above ignore activity characteristics and assume all activities are equally important. Further, there exists a very high dependency on PPC in the industry as its limitations are generally overlooked. PPC not only disregards activity characteristics but also neglects the consideration of successor activities. Consequently, this paper proposes additional metrics to improve weekly work planning and overall workflow. The metrics are designed to complement PPC and other metrics and not to replace them. 


\subsection{Weekly Work Plan Metrics}

Required Level (RL):

RL measures the number of required/critical activities with respect to the number of activities on the weekly work plan. This metric is a natural complement to the CL and PRCO metrics discussed before.

$$
R L=\text { Required Will/Will }
$$

The purpose of RL is to help planners determine the criticality level of their activities and is thus calculated before the WWP starts. A high RL value means that many activities on the WWP are considered critical. Therefore, the team should attempt to complete all activities and obtain a high PPC as well.

Completed Uncommitted (CU):

$\mathrm{CU}$ is a metric that measures work performed that was not on the WWP with respect to the total activities completed. It is important to note that total activities completed here is different from the "Did" in PPC, as backlog and new are not included in PPC calculations.

$$
\begin{gathered}
C U=\frac{\text { Executed }- \text { Executed from Will }}{\text { Did }+ \text { Backlog }+ \text { New }} \\
C U=\frac{\text { Executed from Backlog }+ \text { Executed from New }}{\text { Did }+ \text { Backlog }+ \text { New }}
\end{gathered}
$$

$\mathrm{CU}$ is proposed to address some PPC limitations. PPC does not distinguish between WWP, backlog, and new activities. As a result, an increase in CU can indicate problems in anticipating tasks and in the make ready process.

Figure 1 demonstrates how to calculate RL and CU. The figure shows the number of activities planned and the number of activities executed with respect to their status: Required on WWP, Not Required but on WWP, New, and Backlog. Dividing the number of planned required activities (10) by the total number of planned activities on the WWP (13) gives an RL value of 77\%. On the other hand, dividing the executed New activities (1) + executed Backlog activities (2) by the total number of activities executed (11) results in a CU value of $27 \%$.

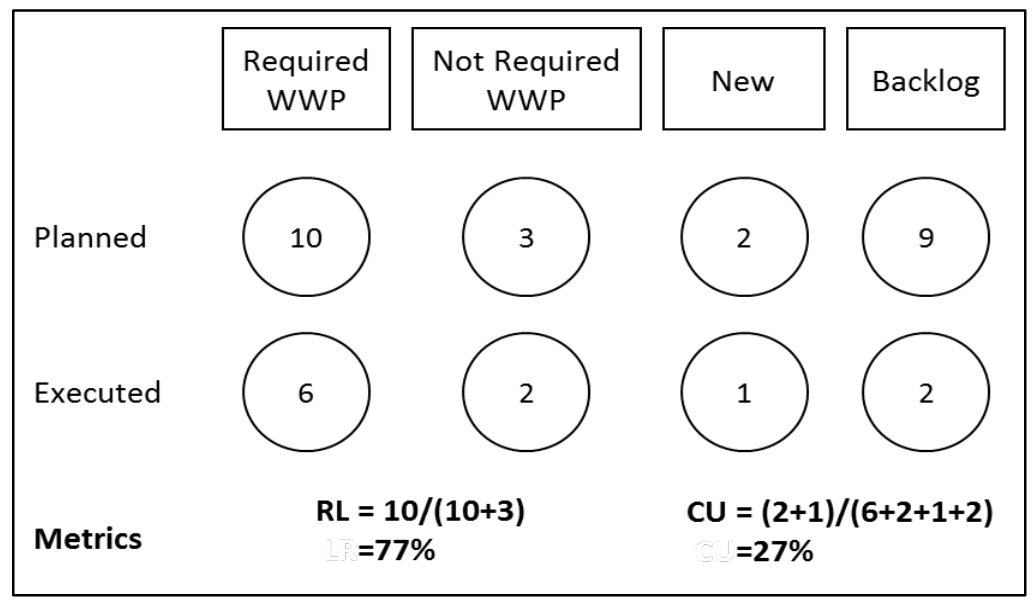

Figure 1. Sample Calculations for RL and CU 


\subsection{Workflow Reliability Metrics}

Labor Hours Reliability Index (LHRI):

LHRI compares the percent of work completed in terms of labor hours with respect to the total amount of expected labor hours. For clarification, two cases are shown below. Both cases show the same ten activities but with different percentages of completion. It is observed that the PPC is easily impacted.

$$
\text { LHRI }=\frac{\% \text { of Work Completed } x \text { Expected Labor hrs }}{\text { Total Expected Labor hrs }}
$$

\begin{tabular}{|l|l|l|l|l|l|l|l|l|l|l|}
\hline Activities (Case 1) & A & B & C & D & E & F & G & H & I & J \\
\hline Labor-hrs of Activity & 100 & 200 & 250 & 150 & 300 & 600 & 400 & 100 & 50 & 50 \\
\hline \% Comp. & $100 \%$ & $50 \%$ & $30 \%$ & $10 \%$ & $80 \%$ & $100 \%$ & $100 \%$ & $50 \%$ & $0 \%$ & $0 \%$ \\
$\qquad P P C=\frac{3}{10}=30 \%$ \\
LHRI $=\frac{(100 \times 1)+(200 * 0.5)+(250 x 0.3) \ldots \ldots}{100+200+250 \ldots \ldots}=\frac{1580}{2200}=71.8 \%$
\end{tabular}

\begin{tabular}{|l|l|l|l|l|l|l|l|l|l|l|}
\hline Activities (Case 2) & A & B & C & D & E & F & G & H & I & J \\
\hline Labor-hrs of Activity & 100 & 200 & 250 & 150 & 300 & 600 & 400 & 100 & 50 & 50 \\
\hline \% Comp. & $100 \%$ & $100 \%$ & $100 \%$ & $100 \%$ & $100 \%$ & $0 \%$ & $100 \%$ & $100 \%$ & $100 \%$ & $100 \%$ \\
\hline
\end{tabular}

$$
\begin{gathered}
P P C=\frac{9}{10}=90 \% \\
L H R I=\frac{(100 \times 1)+(200 * 1)+(250 \times 1) \ldots \ldots}{100+200+250 \ldots \ldots}=\frac{1600}{2200}=72.7 \%
\end{gathered}
$$

As can be inferred from cases 1 and 2, PPC ignores the amount of labor hours an activity needs. This is misleading since not all tasks are of equal value. Case 1 shows that a low PPC and an average LHRI show an average performance. Alternatively, case 2 shows that a high value of PPC can be accompanied with an average value of LHRI indicating an average performance. Accordingly, these metrics can be used together to show a more refined assessment.

Progress Priority (PP):

Progress Priority is based on the time plus sum of sons priority rule. In general, the priority rule ranks any given schedule's activities based on the time required by the activity plus the time required for all activities that succeed it (Khattab and Choobineh 1990). Therefore, PP compares the time of the activities completed in addition to that of their successors with respect to the time of all activities on the WWP that should have been completed in addition to the time of their successors.

$$
P P=\frac{\sum \text { Time Plus Sum of Successors Completed }}{\sum \text { Time Plus Sum of Successors of WWP Should }}
$$

Two cases are shown below to explain the calculation of the PP metric. Calculations are based on the CPM Network shown in Figure 2. Activities shown in red represent activities that were placed on the WWP. 


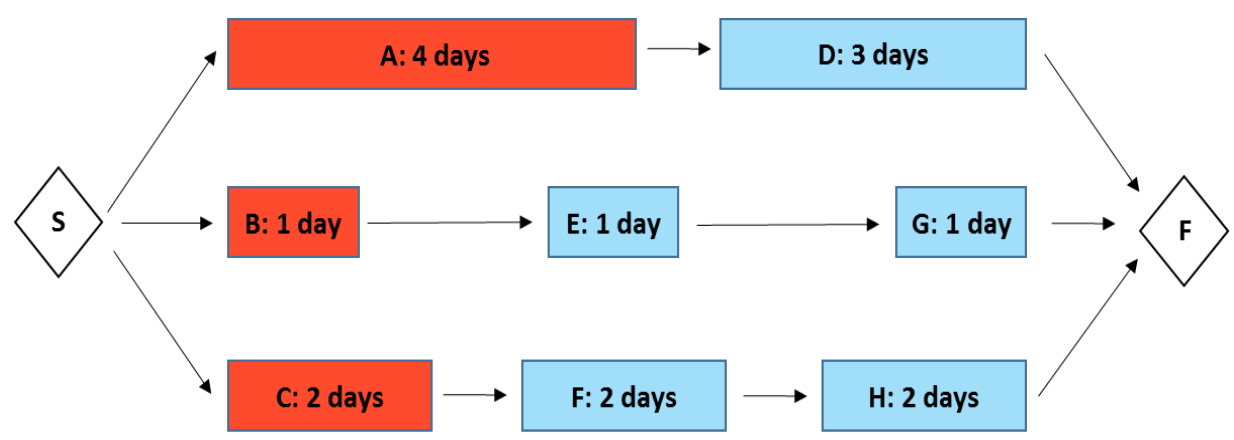

Figure 2: CPM Network

Case 1:

Activities A and C were 100\% completed. However, activity B was not fully completed by the end of the week.

$$
P P C=\frac{2}{3}=66.7 \%, \quad P P=\frac{7+6}{7+3+6}=\frac{13}{16}=81.25 \%
$$

Case 2:

Activities B and C were $100 \%$ completed. However, activity A was not fully completed by the end of the week.

$$
P P C=\frac{2}{3}=66.7 \%, \quad P P=\frac{3+6}{7+3+6}=\frac{9}{16}=56.25 \%
$$

It can be inferred that PP can be different for the same value of PPC. PP can be used to maintain a smooth workflow as it measures the amount of work done and the amount of work opened by the completed activities for downstream tasks compared to the amount of work that was expected to be opened. To ensure a good workflow, the authors recommend looking at the plan as a whole and not on individual activities.

\section{CONCLUSION}

This paper presents an overview of most LPS metrics that have been developed so far. Currently, PPC is the most used metric in the industry. Nevertheless, many metrics have been proposed in the literature to complement PPC. Additional metrics are proposed in this study to address weekly work planning and workflow. RL and CU should be used to complement PPC at the work plan level. RL is a leading indicator to show the percentage of critical tasks on the WWP. CU distinguishes between WWP, backlog, and new activities and measures the amount of work done that was not on the WWP. Moreover, LHRI and PP are suggested as workflow metrics to address the PPC limitations in measuring the volume and amount of work opened, respectively.

This paper highlights the fact that many metrics are currently available and new metrics are being developed. However, there is little research that systematically applies those metrics to identify their predictive power in isolation or in combination. Research is difficult to conduct because of how teams document LPS. The advent of database-driven LPS tools provides an opportunity for the systematic analysis of the LPS metrics. Research can help advance how LPS tools are implemented and the resulting organized datasets can advance research by providing well-organized and structured datasets for further analysis. In this context, the authors are in the process of applying these metrics on actual projects to assess their utility and highlight major issues in production planning. 


\section{REFERENCES}

Alarcón, L. F., Salvatierra, J. L. \& Letelier, J. A. (2014). Using Last Planner Indicators to Identify Early Signs of Project Performance. In: 22nd Annual Conference of the International Group for Lean Construction. Oslo, Norway, pp. 547-558.

Ballard, G. (1997). Lookahead Planning: The Missing Link in Production Control. In: 5th Annual Conference of the International Group for Lean Construction. Gold Coast, Australia, pp. 13-26.

Ballard, G. (2000). The Last Planner System of Production Control. Ph. D. Dissertation, Faculty of Eng., School of Civil Eng., The University of Birmingham, UK, 192 pp.

Chitla, V. R. and Abdelhamid, T. S. (2003). Comparing Process Improvement Initiatives Based on Percent Plan Complete and Labour Utilization Factors. In: 11th Annual Conference of the International Group for Lean Construction. Virginia, USA.

Emdanat, S. and Azambuja, M. (2016). Aligning Near and Long Term Planning for LPS Implementations: A Review of Existing and New Metrics. In: 24th Annual Conference of the International Group for Lean Construction. Boston, USA.

Gonzalez, V., Alarcon, L. F., and Mundaca, F. (2008). Investigating the Relationship between Planning Reliability and Project Performance. Production Planning \& Control, 19(5), pp. 461-474.

Hamzeh, F. R. \& Aridi, O. Z. (2013). Modeling the Last Planner System Metrics: A Case Study of an Aec Company. In: 21th Annual Conference of the International Group for Lean Construction. Fortaleza, Brazil, pp. 599-608.

Hamzeh, F.R., Ballard, G., and Tommelein, I.D. (2008). Improving Construction Workflow-The Connective Role of Lookahead Planning. In: 16th Annual Conference of the International Group for Lean Construction. Manchester, UK, pp. 635-646.

Hamzeh, F. R., Saab, I., Tommelein, I. D., and Ballard, G. (2015a). Understanding the Role of "Tasks Anticipated" in Lookahead Planning through Simulation. Automation in Construction, 49, Part A, pp. 18-26.

Hamzeh, F. R., Zankoul, E., and Rouhana, C. (2015b). How can 'Tasks Made Ready' during Lookahead Planning Impact Reliable Workflow and Project Duration? Construction Management and Economics, 33(4), pp. 243-258.

Jang, J. W., and Kim, Y. W. (2007). Use of Percent of Constraint Removal to Measure the Make Ready Process. In: 15th Annual Conference of the International Group for Lean Construction. East Lansing, Michigan, USA. 15, pp. 529-38.

Jang, J. W., and Kim, Y. W. (2008). The Relationship between the Make-Ready Process and Project Schedule Performance. In: 16th Annual Conference of the International Group for Lean Construction. Manchester, UK. 16, pp. 647-56.

Khattab, M. and Choobineh, F. (1990). A New Heuristic for Project Scheduling with a Single Resource Constraint. Computers \& Industrial Engineering, 19(1), pp. 514-518.

Mitropoulos, P. T. (2005). Planned Work Ready: A Proactive Metric for Project Control. In: 13th Annual Conference of the International Group for Lean Construction. Sydney, Australia.

Priven, V., Sacks, R., Seppänen, O., and Savosnick, J. (2014). A Lean Workflow Index for Construction Projects. In: 22nd Annual Conference of the International Group for Lean Construction. Oslo, Norway.

Rouhana, C. and Hamzeh, F. (2016). An ABC Approach to Modeling the Emergence of 'New Tasks' in Weekly Construction Planning. Lean Construction Journal, pp.35-56.

Tommelein, I. D., and Ballard, G. (1997). Look-ahead Planning: Screening and Pulling. In: Seminário Internacional sobre Lean Construction, 2. Sao Paulo, Brazil. 